\title{
Rapid Reconfiguration of Paediatric Services in a District General Hospital During COVID-19, Addressing Challenges, and Seeing Opportunities
}

\author{
Authors: \\ Ahmed Faraz Khan, ${ }^{1}$ Julie McConville, ${ }^{2}{ }^{*}$ Suzanne Barr ${ }^{3}$ \\ 1. Daisy Hill Hospital, Children and Young People Services, Southern Health \& Social \\ Care Trust, Newry, Northern Ireland \\ 2. Specialist Child Health and Disabilities, Southern Health \& Social Care Trust, \\ Craigavon, Northern Ireland \\ 3. Medical Directorate, Southern Health \& Social Care Trust, Craigavon Area Hospital, \\ Craigavon, Northern Ireland \\ *Correspondence to Suzanne.Barr@southerntrust.hscni.net
}

Disclosure: $\quad$ The authors have declared no conflicts of interest.

Received:

Accepted:

Keywords:

Citation:
22.09 .20

21.12 .20

Accessibility, coronavirus disease (COVID-19), innovation, opportunities, technology, together, waiting list, whole team approach.

EMJ Innov. 2021;5[1]:82-87.

\section{Abstract}

The scale, speed, and impact of the coronavirus disease (COVID-19) pandemic disruption to healthcare services has been unprecedented, placing significant additional pressures on the National Health Service (NHS). COVID-19 presented exceptional challenges to vulnerable families and is placing increasing pressure on children's services. The child population does not seem to have been severely impacted by COVID-19; however, some will require hospital care in addition to the current caseload. It is imperative that steps are taken to ensure continued delivery of urgent and emergency paediatric services and the associated maternity and neonatal services at local levels throughout the pandemic. A rapid reconfiguration of services was necessary when the pandemic reached the NHS. Healthcare services had to rethink how to deliver care in the short and medium term, better preparing them for future demands and ensuring that safe and effective care was maintained.

\section{INTRODUCTION}

The Southern Health and Social Care Trust (SHSCT) has two acute hospitals: Craigavon Area Hospital (CAH) and Daisy Hill Hospital $(\mathrm{DHH})$. Both hospitals deliver emergency department services and inpatient services for medical, surgical, paediatrics, obstetrician and gynaecology, and radiology departments, laboratory, and other support services. The paediatric ward in $\mathrm{DHH}$ has 13 inpatient beds and cots, predominantly single room, six elective surgical beds, a paediatric theatre, and four single

rooms for Short Stay Paediatric Assessment Unit (SSPAU). There are an additional six cots in the Special Care Baby Unit (SCBU), which serves around 2,000 deliveries in the adjacent maternity unit each year. There is a three-tier medical rota with appropriate levels of nursing staff monitoring all shifts ensuring high-quality care. The SSAPU opens at 9 a.m. to 10 p.m. on weekdays, and receives referrals from primary care teams and supports the emergency department. In addition to this, the team also provides telephone advice via a paediatric advice line (PAL) to primary care teams and service users. 


\section{RECONFIGURATION}

"The best defence against any outbreak is a strong health system," stated the DirectorGeneral of the World Health Organization (WHO), Tedros Adhanhom Ghebreyesus, teaching the importance of exploring healthcare services and further strengthening them through necessary adaptations in the face of the pandemic. During the first wave of the coronavirus disease (COVID-19) pandemic, $\mathrm{CAH}$ was designated as a COVID-19 hospital site and $\mathrm{DHH}$ as a non-COVID-19 site. Some resources had to be relocated from $\mathrm{DHH}$ to the site of $\mathrm{CAH}$; this included the temporary closure of the $\mathrm{DHH}$ emergency department, resulting in the greatest impact of service reconfiguration initially experienced by $\mathrm{DHH}$.

Paediatric services in $\mathrm{DHH}$ commenced a rapid reconfiguration as per advice from Northern Ireland Child Health Partnership forum and COVID-19 Silver Command Centre. This was to support adult medical services because of increasing numbers of adult patients. Service delivery had to be proactive, responsive, and focussed on safe and effective patient care and staff wellbeing. A service improvement approach was adopted so that any changes made would be reviewed and acted upon, giving a continuous cycle of improvement and opportunity throughout the pandemic, recognising the benefits and challenges that lay ahead of the service: "Certain factors may help to foster an environment that is conducive to change and improvement. An organisation where there is strong leadership and everyone is focussed on improving patient care is more likely to develop motivated staff with a desire for continuous quality improvement," as stated in NHS Improving Quality guidelines. ${ }^{2}$

Any temporary reconfiguration of paediatric services had to ensure that (Table 1):

> Steps to mitigate the impact on vital children's services were taken while services responded to the pandemic.

$>$ Reconfiguration was to be completed within 1 week.

> Children with non-COVID-19 conditions who required urgent and emergency care continued to receive appropriate general and specialist hospital care.
> Agreed clinical pathways were in place to ensure appropriate, timely, and safe care.

> Paediatric cover was in place to support obstetric services and neonatal units.

\section{OPPORTUNITIES AND LESSONS LEARNT}

\section{Whole System Approach}

The necessity and speed of the reconfiguration allowed recognition of a common, greater goal across regional, primary, and secondary care. Streamlined care pathways were agreed through positive and meaningful multidisciplinary engagements. Through positive engagement and working together, the COVID-19 pandemic broke down barriers and refocussed services. Collaborative working between primary and secondary care improved; these relationships still need nurturing and support to enhance shared learning. Such an approach can be effective in many settings and institutions throughout public services and healthcare, as long as there is a sense of a common goal and shared purpose.

\section{Flexible Working Staff: Coming Together}

Flexible working arrangements were afforded to staff to work onsite and offsite, with some virtual consultations completed offsite. Computer and technological support were crucial during this process. Staffing reductions from redeployment would have caused the middle- and junior-tier rotas to have been harder to fulfil without staff coming together with some possible solutions. Their active role in the process enabled solutions to be found rather than furthered challenges faced by the service. Consultants delivered onsite cover for the middle-tier rota, which provided shared responsibility. Skills, experience, and expertise were spread across the rota, ensuring safe and effective care of patients. The flexible working arrangements were beneficial in the shorter term; however, to be sustained in the long term, further exploration is required with regard to productivity and service delivery. As part of a service improvement cycle, this adaptation will continue to be reviewed and evidenced, ensuring that the needs of the service can be met and sustained. 
Table 1: Reconfiguration of paediatric services, the challenges, and outcomes.

A

\section{Structural and patient flow changes}

\section{Challenges}

Maintaining communication with service users and the wider healthcare system in the hospital, primary care teams, and regionally.

The inpatient ward in $\mathrm{DHH}$ to close for all new paediatric patient admissions from $3^{\text {rd }}$ April 2020.

An arrangement for streamlined patient care pathways was essential for the access to appropriate care for service users.

DHH ED was temporarily closed to strengthen CAH ED staffing levels; therefore, temporarily, there was no ED cover available in $\mathrm{DHH}$.

\section{Actions taken to mitigate the risk}

A multidisciplinary team that included clinical and operational staff was formed to implement the rapid reconfiguration. It was a two-pronged approach including local and regional considerations to ensure effectiveness. Mechanisms for transparent and meaningful communication and collaboration were commenced and maintained throughout the pandemic, not only within the Southern Trust but also with other regional services.

Daily operational meetings were convened, ensuring that there were open and clear lines of communication, with opportunities for services across the organisation to support one another in their pressures, as well as learn from one another.

A regional approach to triggering, monitoring, and communication was agreed by the regional Child Health Partnership Forum to ensure the safe and effective care of patients across the region. This agreement allowed for consistency and collaborative working across all Trusts so that all children in need received the same high standard of care regardless of which area they resided in.

NMS forum established. This forum included paediatric and GP representatives to improve shared care responsibilities and meaningful communication through active stakeholder engagement.

The patient care pathway from primary care to hospital paediatric services was discussed and developed so that barriers were identified and addressed where possible. This enabled opportunities to tackle any delaying areas and work together to find resolutions, which facilitated more effective care provision. These were continuously reviewed and refined over the following months, allowing for continuous improvement to occur.

The patient flow pathway was adapted to address the deficiency of no ED and no inpatient ward in DHH. By doing so, there was a clear channel of how care provision would continue to be delivered without these components on site.

As the inpatient unit was closed for paediatric services, all remaining inpatients in $\mathrm{DHH}$ either transferred to $\mathrm{CAH}$ or discharged home with appropriate follow-up arrangements.

The inpatient ward was handed over to adult services to utilise space for the safe and effective care of adult patients during the COVID-19 pandemic. A sense of togetherness was reinforced between colleagues as paediatric services supported the pressures on adult services through this change.

Paediatric services were changed to SSPAU/ambulatory-based services. Clinical management was mainly delivered through the SSPAU and phone consultations to meet the needs of the patient.

Paediatric phone clinics started to utilise the virtual consultation platform, allowing for continued care of patients, reduction of waiting lists, and enhanced communication and relationship building with service users and their families. Virtual consultation operating procedures were developed so that there were clear protocols and guidelines for all users. Staff training was provided where necessary so that everyone was equipped with the skills needed to utilise this tool.

A paediatric resuscitation area was created within SSPAU for unexpected, unwell patients so that there was a contingency in place for all eventualities of patient safety measures in the absence of ED in DHH.

SIM training sessions were delivered for all staff for the management of sick children.

All ambulances to $\mathrm{DHH}$ were diverted to $\mathrm{CAH}$. This arrangement was agreed after close consultation with Northern Ireland ambulance services; again, strengthening relationships and highlighting the importance of partnership working

CAH: Craigavon Area Hospital; DHH: Daisy Hill Hospital; ED: emergency department; GP: general practitioner; NMS: No More Silo; SIM: simulatory; SSPAU: Short Stay Paediatric Assessment unit. 


\section{Staffing Changes}

\section{Challenges}

A number of paediatric staff were redeployed to CAH acute medical department, therefore paediatric services had to manage patients with reduced staffing levels.

Anxiety levels among staff were particularly heightened due to professional and personal pressures faced throughout the pandemic. With changes to their workplace, routines, and a rapid reconfiguration to their service, some staff found themselves feeling additionally stressed.

\section{Actions taken to mitigate the risk}

Regular staff meetings were established to communicate with and listen to front-line staff and enable them to safely voice their concerns where appropriate, providing reassurance, guidance, and support under the strains faced in the pandemic. Front-line staff were active participants in the reconfiguration, keeping them involved and informed.

Working patterns were changed to allow safer staffing levels. This included collapsing three-tier rotas into two-tier rotas on $\mathrm{DHH}$ site. This was entirely new to the service and increased the sense of togetherness and teamworking in the face of adversity.

Consultants delivered onsite resident duties, which added to the sense of a shared workload in an already challenging environment. Consultants also completed middle-grade/registrar duties, providing senior decision-making at the front end of the services.

Some consultant staff offered their assistance to adult medical services, enhancing a sense of unity and togetherness across the organisation.

Given the potential risk posed to safe practice from potential reduced staffing levels as a consequence of sickness and self-isolation, a backup consultant rota was established for out-of-hours consultant shifts. This contingency offered assurance to the service when necessary.

Organisational staff support. Occupational health staff support sessions and psychological sessions were offered to all staff across the organisation, and a staff newsletter was provided regularly to all staff throughout the Trust. This was to offer health and wellbeing supports as well as a sense of value within the organisation.

CAH: Craigavon Area Hospital; DHH: Daisy Hill Hospital.

\section{Risk management}

\section{Challenges}

Clinical risk was higher because of rapid reconfiguration of services; therefore, it was vital to closely monitor risk management processes alongside any actions taken, which are noted within the table.

\section{Actions taken to mitigate the risk}

The senior management team held regular operational meetings to share information and provide updates.

Patient flow was monitored daily in MDT meetings to identify and address any areas of delay and note areas of progress.

Clinical incidents were closely monitored, and any learning disseminated throughout MDT engagement and clinical governance/patient safety forums

Virtual patient safety and mortality and morbidity meetings started via Zoom (San Jose, California, USA).

An MDT handover commenced 3 times per day, every day. This incorporated patient safety briefs, which included unusual patient presentations, any patient on unusual medication, on intravenous fluids, same-name patients, any expected transfers, and any clinical incidents during the previous 8 hours. This utilised the handover communication tool for further enhancing patient care and assisting with decision-making: "Decisions should be individualised - this means that decisions must take into account patient's individual characteristics, preferences, and prognosis." 4

Weekly operational/clinical group meetings were established to review and plan for the following week, allowing for forward planning and reducing the risk through a structured approach.

A paediatric and GP interface forum convened to discuss and mitigate any patient flow risks.

Daily regional paediatrics network forum meetings took place, giving a platform for shared learning across the region, assisting with mitigating any risks faced and creating an environment for safe learning even during a pandemic. 


\section{Innovation Using Technology}

Innovative work can offer organisations new ways of addressing current and future challenges, reaching their targets, and addressing backlogs, which may not have been achievable previously. Because of the current pandemic, face-to-face clinics and interactions decreased, replaced by virtual consultations and meetings, allowing for staff time to be more structured and focussed.

Virtual consultations were implemented, ensuring that patient medical needs continued to be met within a safe and controlled environment. The more effective use of resources had a positive impact on the clinic waiting lists. The PAL was extended from 9 a.m. to 10 p.m. and 4 hours on weekends with a senior decision-maker available to provide advice to primary care teams and service users.

\section{Remote Access and Clinical Application}

The current pandemic highlighted the requirement for clinical teams to have remote electronic access of resources. Innovative technology is being enthusiastically progressed within the service by the further development of a paediatric smart device app to enable remote information accessibility of clinical guidelines, care pathways, and services contact directory. Appropriate protocols and guidelines were developed for all when using these technologies so that this could become embedded in the future of service delivery, also keeping within organisational governance. With emerging evidence on the persistence of coronavirus on inanimate objects such as shared computers in the patient care environment, the app, available on personal devices, provides an alternative access point for information. The ambition is to roll this app out to both primary care teams and secondary paediatric services following a successful testing period. This in turn will continue to maintain and sustain communication between services and to improve care pathways.

\section{Service User Engagement}

To ensure service user engagement throughout the reconfiguration and adaptations, patient feedback was actively sought so that all stakeholders, not just those multidisciplinary partners, were consulted with. Those who received appointments additionally received a leaflet explaining the changes being made to the service. This information was also made available on the app as a full consultation with an explanation as to why service could not be commenced, attributed to the timescales involved in the reconfiguration: "Coproductive working relationship with children, care leavers, their families, and carers to establish what matters to them and to ensure they feel respected and informed. This includes explaining to children and families the ways in which the COVID-19 arrangements may impact on the provision of their care and support." 3 In recognition of patients being active stakeholders, a concerted effort to collect feedback was made to assist the shaping of future service delivery. Service users who attended the paediatric services during this time were sent feedback forms to provide input into the changes that were being made. This allowed service users to feel like they were active participants, and gain greater understanding of the restraints being put upon the healthcare system and the rationale for the reconfiguration. Service users were extremely positive about the increased sense of accessibility for advice, information, and care through the use of the PAL, the app, and virtual consultations. Families who would have struggled to arrange time off work, childcare, or travel arrangements to accommodate consultation had now been given the freedom of a less stressful appointment through the facilitation of virtual consultations. The service user feedback data collected will be collated and analysed in coming months to allow for shared learning with senior management team to convey the work achieved by the service and the value in learning from the opportunities identified throughout the pandemic. This piece of work will then be further shared across the Trust through the 'Learning from Experience' forum, which is attended by all directorates, facilitating further dissemination of the learning and a chance to reflect on the sense of achievement of overcoming the challenges faced.

Feedback and ongoing collaboration with service users can be repeated in any setting as long as there is honest and transparent communication and expectations are managed appropriately. The demand on services remains the same; however, the pandemic allowed the freedom to 
make innovative progress in a safe, productive, and efficient manner with all stakeholders working together for the greater good.

\section{THE WAY FORWARD}

There is more that can be done when working in a whole system approach. The authors will continue to explore innovative solutions to improve patient outcomes and drive efficiency. This case study proved the value of taking measured, brave decisions to try new innovative methods, and taking a step back to reflect upon how best to provide safe, high-quality services using a collective and collaborative approach during a pandemic. Communication on every level with all active participants allowed for greater understanding, better informed decision-making, and reduced risk. The key themes of meaningful engagement, positive communication, teamwork, and support can be adopted into any organisation and any setting, developing a culture of continuous improvement, and ultimately service reward for the benefit of all.

\section{References}

1. World Health Organization (WHO). WHO releases guidelines to help countries maintain essential health services during the COVID-19 pandemic. 2020. Available at: https://www.who.int/news-room/ detail/30-03-2020-who-releasesguidelines-to-help-countriesmaintain-essential-health-servicesduring-the-covid-19-pandemic. Last accessed: 21 December 2020.
2. NHS. First steps towards quality improvement: a simple guide to improving services. 2014. Available at: https://www.england.nhs.uk/ improvement-hub/wp-content/ uploads/sites/44/2011/06/service_ improvement_guide_2014.pdf. Last accessed: 21 December 2020.

3. Welsh Government. Children's social services during the COVID-19 pandemic: guidance. 2020. Available at: https://gov.wales/childrens-social- services-during-covid-19-pandemicguidance-html. Last accessed: 21 December 2020

4. The Royal College of Paediatrics and Child Health (RCPCH). Ethics framework for use in acute paediatric settings during COVID-19 pandemic. 2020. Available at: https://www.rcpch ac.uk/resources/ethics-frameworkuse-acute-paediatric-settings-duringcovid-19-pandemic. Last accessed: 21 December 2020. 